\title{
AES Proceedings
}

\author{
Annual Meeting of the American Epilepsy Society
}

\section{December 3, 2005 Investigators' Workshop 2:30 p.m.-5:45 p.m.}

\author{
MIW.001 \\ Seizures Beget Seizures \\ Yehezkel Ben-Ari (INMED Institute, Marseilles, France)
}

Seizures produce long lasting alterations of the networks that lead to a reduction of the threshold of further seizures. In adults, the underlying mechanisms have been extensively investigated in particular in temporal lobe epilepsies. Seizures notably produced by kainate or pilocarpine induce cell death in vulnerable regions of the hippocampus. This is followed by sprouting and the formation of novel glutamatergic synapses - notably mossy fibres of CA3 pyramidal neurons. These new synapses are functional as attested by the massive rise of glutamatergic EPSCs in target epileptic neurons. Interestingly also recordings from temporal lobe epileptic animals, reveal that these synapses are aberrant since control granule cells use only AMPA receptors mediated synapses whereas after the formation of novel synapses, most EPScs are purely kainate receptor mediated. This implies directly that the sprouting has induced the formation of novel synapses that operate differently from the control ones. Other studies have shown also that the inaugurating status epilepticus induced by the injections of the convulsive agent triggers a cascade of events associated with the activation of hundreds of genes in the hours -days that follow the seizures that are responsible for the sprouting and neo-synapse formation. The period of roughly 3 weeks that follows the seizures is a "silent" period during which seizures are not generally generated and spontaneous events do not take place. Ed Dudek will deal with the events that occur at that stage. One additional basic issue concerns the generation by thalamo- cortical connections of fst oscillations and the role of GABAergic synapses in that event. Mircea Steriade will summarize these events and explain how seizures are generated in the neocortex and how they can affect the network. Finally, relying on the triple chamber that accommodate the intact neonatal hippocampi and their connections, Y Ben-Ari will describe the mechanisms of seizures beget seizures during development. In essence, the questions here are which seizures beget seizures and produce long lasting alterations and which do not. This has important implications as to the determination of the mechanisms at work but also may be useful in a clinical perspective to evaluate potentially deleterious seizures.

\section{MIW.002}

Fly, Fish and Worm Models of Epilepsy

${ }^{3}$ Guy Caldwell, ${ }^{2}$ Mark Tanouye, and ${ }^{1}$ Scott C. Baraban $\left({ }^{1}\right.$ Neurological Surgery, UCSF, San Francisco, CA $;{ }^{2}$ Environmental Science, UC Berkeley, Berkeley, CA; and ${ }^{3}$ Biological Sciences, University of Alabama, Tuscaloosa, AL)

Rodent models of experimental epilepsy have been an especially valuable aid in understanding fundamental aspects of human seizure disorders. While there are distinct advantages to using a rodent model of a human neurological disorder, there is no rationale to support our almost exclusive reliance on this species. Indeed, fundamental research related to genetic modifiers of epilepsy, high-throughput anticonvulsant drug screening and forward-genetic screening strategies to uncover novel epilepsy genes are better suited to simpler systems. Exciting new discoveries in the general field of neurobiology have begun to exploit the experimental advantages of simpler organisms such as C. elegans (worms), Drosophila melanogaster (fruit flies) and Danio rerio (zebrafish). Similar discoveries could be possible in the epilepsy field. To highlight recent advances, an Investigator Workshop is planned to present the current state of knowledge in these systems (and provide a forum to discuss where we can, or should, go from here). Guy Caldwell (University of Alabama) will discuss his work with C.elegans lissencephaly mutants. Using a pentylenetetrazole exposure paradigm, they have uncovered a convulsive phenotype that correlated with interesting intraneuronal deficits in presynaptic GABA vesicle trafficking. This suggests it may be possible to separate the intrinsic neuronal deficits leading to LIS1-dependent epilepsy from the more overt cortical defects associated with neuronal migration. Mark Tanouye (UC Berkeley) will present studies on mutant and wild-type Drosophila tested in a stimulation-induced seizure protocol. Using this approach they have identified "epilepsy" mutants that are especially prone to seizures when compared with normal flies and have begun to explore seizure-suppressor and seizure-enhancer mutations. Scott C. Baraban (UCSF) will discuss his work with zebrafish larvae. Using a PTZ exposure protocol, they have described the electrophysiological, behavioral, pharmacological and molecular aspects of a novel simple vertebrate seizure model. Screening a colony of over 6300 ENU-mutagenized zebrafish, seizure-resistant larvae were identified and are now undergoing further characterization and gene mapping. Jeffrey L. Noebels (Baylor) will act as moderator for a lively discussion of these topics.

\section{MIW.003}

Imaging Excitatory Neurotransmission

${ }^{1}$ Jonathan Wetherington, ${ }^{2}$ Ognen Petroff, and ${ }^{3}$ Matthias Koepp $\left({ }^{1}\right.$ Dept of Pharmacology, Emory University, Atlanta, $\mathrm{AL} ;{ }^{2}$ Dept of Neurology, Yale University, New Haven, CT; and ${ }^{3}$ Dept of Clinical and Experimental Epilepsy, Institute of Neurology, UCL, London, United Kingdom)

The N-methyl-D-aspartate (NMDA) ion channel plays a role in neuroprotection, neurodegeneration, long-term potentiation, memory, and cognition. It is implicated in the pathophysiology of several neurologi$\mathrm{cal}$ and neuropsychiatric conditions. The development of effective radiotracers for the study of NMDA receptors is critical for our understanding of their function, and their modulation by endogenous substances or therapeutic drugs. The intrachannel PCP binding site has attracted most attention, as it is only accessible when the channel is in the active and "open" state", but not when it is in the inactive or "closed" state. The physical location of the NMDA/PCP receptor not only makes it an important theoretical imaging target, but also complicates the development of suitable PET and SPECT radiotracers for this site and attempts to quantify in-vivo binding. An intimate understanding of the biochemical, pharmacological, physiological and behavioral processes associated with the NMDA ion channel is essential to develop improved imaging agents and interpret in-vivo measurements.

This workshop will focus on the development of creative approaches to the study of excitatory neurotransmission in patients with epilepsy using MRI/MRS, PET or SPECT. It will provide participants with an understanding of the biochemical, pharmacological, physiological and behavioral processes associated with the NMDA ion channel and an insight into the difficulties and complexities of imaging excitatory neurotransmission in vivo. The participants of this workshop will discuss the animal and pharmacological models used for in-vitro and in vivo assessment of NMDA receptor functions.

The multi-disciplinary nature of this workshop provides opportunities for interactions between participants and faculty with diverse backgrounds including paediatric and adult neurology/epileptology, basic neuroscience, pharmacology, neurophysiology, neuroradiology and nuclear medicine. 
Results: Patients $(\mathrm{N}=104)$ were randomized to rufinamide $(\mathrm{n}=$ $52)$ or placebo $(\mathrm{n}=52)$. The mean daily dose of rufinamide during DB was $2970.7 \mathrm{mg} / \mathrm{d}$. Rufinamide-treated patients demonstrated a significantly greater median number of days to meet $\geq 1$ exit criteria versus placebo-treated patients ( 4.8 vs 2.4 respectively; $p=0.0499$ ). Approximately the same percentage of patients in the rufinamide group $(67.3 \%)$ as in the placebo group $(69.2 \%)$ met 1 exit criterion ( $\mathrm{p}=$ 0.8809 ). The median times to $1^{\text {st }}, 2^{\text {nd }}$, and $3^{\text {rd }}$ partial seizures were significantly greater with rufinamide than with placebo $(p \leq 0.0348)$ The most commonly reported adverse events (AEs) $(\geq 10 \%)$ for both groups were somnolence, nausea, and headache. Clinically relevant abnormal laboratory values were noted in 9 rufinamide-treated patients for alkaline phosphatase, SGOT, SGPT, uric acid, potassium, eosinophils, and WBCs. A clinically notable decrease in systolic blood pressure was observed in 2 rufinamide-treated patients (3.9\%). Nonfatal serious AEs occurred in only 1 rufinamide-treated patient. Three rufinamideand 2 placebo-treated patients discontinued due to nonserious AEs.

Conclusions: Rufinamide was significantly more effective than placebo as monotherapy for the treatment of refractory partial seizures as demonstrated by the primary efficacy results. Rufinamide was well tolerated, with the majority of AEs mild to moderate in severity. (Supported by Eisai Inc.)

2.259

SAFETY OF RAPID INTRAVENOUS LOADING OF UNDILUTED VALPROIC ACID (VPA)

Nita A. Limdi, Robert Knowlton, Edward R. Faught, A. LeBron Paige, and Larry Verhoeff (Neurology, University of Alabama at Birmingham, Birmingham, AL)

Rationale: Introduction of IV VPA has allowed its use when oral administration is not feasible. However, the recommended administration rate of $20 \mathrm{mg} /$ minute does not facilitate its use in emergent situations. We present data on safety of IV administration of loading doses (20 or $30 \mathrm{mg} / \mathrm{kg}$ ) of undiluted VPA at $10 \mathrm{mg} / \mathrm{kg} / \mathrm{min}$.

Methods: Patients meeting inclusion criteria were enrolled in this prospective study. Patients in status epilepticus, with hepatic dysfunction, allergy to VPA or lamotrigine therapy were excluded.

VPA loading doses of 20 or $30 \mathrm{mg} / \mathrm{kg}$ were administered undiluted via peripheral IV at $10 \mathrm{mg} / \mathrm{kg} / \mathrm{min}$. Local tolerance was evaluated using the pain scale $(0-10)$. Blood pressure (MAP), heart rate (HR), respiratory rate $(\mathrm{RR})$, and electrocardiogram (EKG) were monitored every $2.5 \mathrm{~min}-$ utes for the first 20 minutes, then at 30, 45 and 60 minutes post-infusion. Level of consciousness (LOC) was evaluated prior to and 60 minutes post-infusion using the NIH Stroke Scale-LOC tool. Total and unbound VPA levels were measured at baseline, 30, 60 minutes, and then 4 hours post-infusion.

Repeated measures ANOVA was used to evaluate the influence of VPA dose on cardiovascular parameters.

Results: Seventeen patients ( 10 men) were enrolled with eight patients receiving $20 \mathrm{mg} / \mathrm{kg}$ and nine $30 \mathrm{mg} / \mathrm{kg}$ loading doses of VPA. There were no differences in age, gender distribution, or weight between the 2 dose groups.

Local tolerance: Patients tolerated the infusion well. Most reports were of minor or no intolerance. Three patients reported severe pain (score $>7 / 10$ ) and two severe cold sensation/numbness (score $>7 / 10$ ). This intolerance was transient, did not require slowing infusion, \& resolved spontaneously in all patients within 3 minutes. There was no difference in duration of intolerance by dose $(\mathrm{p}=0.24)$.

Systemic Tolerance: The cardiovascular tolerance of rapid infusion was good with no significant alteration in LOC, EKG or respiratory parameters. Since the heart rate (HR) in patients receiving $20 \mathrm{mg} / \mathrm{kg}$ was higher than those receiving $30 \mathrm{mg} / \mathrm{kg}$ at baseline $(\mathrm{p}=0.002)$, the repeated measures model ANOVA considered baseline HR and MAP as predictor variables.

Neither time of HR measurement or dose had a significant affect on HR ( $>0.50)$.

Both time of MAP measurement and dose had a significant effect on MAP $(\mathrm{p}<0.05)$. MAP was lower by $2.86 \mathrm{mmHg}$ [95\% CI 0.105 , 5.63] for patients receiving $30 \mathrm{mg} / \mathrm{kg}$ dose than for those receiving 20 $\mathrm{mg} / \mathrm{kg}$ doses. Although this finding is statistically significant, its clinical significance is equivocal.
Pharmacokinetics: Both free \& total VPA concentrations were significanlty higher in the $30 \mathrm{mg} / \mathrm{kg}$ dose group than in the $20 \mathrm{mg} / \mathrm{kg}$ group at $30 \& 60$ minutes $(\mathrm{p}<0.0001)$ but not at 4 hours.

Conclusions: Administration of loading doses (20 or $30 \mathrm{mg} / \mathrm{kg}$ ) of undiluted VPA at $10 \mathrm{mg} / \mathrm{kg} / \mathrm{min}$ is well tolerated with expected peak concentrations over $100 \mathrm{mcg} / \mathrm{ml}$. (Supported in part by Abbott Laboratories under FDA IND\# 62165.)

\subsection{0}

CARBAMAZEPINE MAY INDUCE OSTEOPOROSIS BY INCREASING BONE TURNOVER IN POSTMENOPAUSAL WOMEN

${ }^{1}$ Merete A. Lyngstad Brechan, ${ }^{1}$ Erik Taubøll, ${ }^{2}$ Rune Jemtland, ${ }^{2}$ Kristin Godang, ${ }^{2}$ Jens Bollerslev, and ${ }^{1}$ Leif Gjerstad $\left({ }^{1}\right.$ Department of Neurology, Rikshospitalet University Hospital, Oslo, Norway; and ${ }^{2}$ Department of Medicine, Endocrine Section, Rikshospitalet University Hospital, Oslo, Norway)

Rationale: Several antiepileptic drugs (AEDs) have been associated with disorders of bone mineral metabolism, in particular liver enzyme-inducing AEDs including carbamazepine (CBZ). In addition, postmenopausal women are a vulnerable population for bone loss due to estrogen deficiency. We therefore wanted to study the effect of chronic CBZ treatment in a group of postmenopausal women and to compare the results with individually age-matched controls to reduce possible confounding factors regarding age and lifestyle.

Methods: 13 postmenopausal women with epilepsy on CBZ monotherapy for $>4$ years and 13 age-matched controls consisting of close female friends of the patients were studied. Blood samples were drawn and serum analyzed for indices of bone metabolism including calcium, phosphate, vitamin D (S-25(OH)Vit D), PTH and thyroid hormones. In addition, bone formation markers including alkaline phosphatase (ALP), bone-specific alkaline phosphatase (bALP) and osteocalcin, and CrossLaps (CTX-1) as a marker for bone resorption were analyzed.

Results: ALP and bALP were significantly higher in CBZ treated patients versus controls $(61.4 \mathrm{U} / 1$ and $22.0 \mathrm{U} / 1$ in controls versus 93.2 $\mathrm{U} / 1$ and $33.4 \mathrm{U} / \mathrm{l}$ in CBZ treated women, respectively). Both osteocalcin and CrossLaps were higher in the CBZ treated, although this was not statistically significant $(0.95 \mathrm{nmol} / 1$ and $0.47 \mathrm{mg} / \mathrm{ml}$ in controls compared to $1.13 \mathrm{nmol} / 1$ and $0.58 \mathrm{ng} / \mathrm{ml}$ in CBZ treated, respectively). Vitamin $\mathrm{D}$ was lower in CBZ treated patients vs. controls $(67.4 \mathrm{vs} 97.7 \mathrm{nmol} / \mathrm{l}, \mathrm{p}=$ 0.039 ) as was PTH (3.8 vs $5.2 \mathrm{pmol} / 1, \mathrm{p}=0.28$ ). Serum calcium, ionized calcium and phosphor were the same in both groups. Free thyroxine level was slightly, but significantly lower in CBZ treated women.

Conclusions: The increase in BALP and ALP indicates an effect of CBZ on bone formation, possibly related to an increased bone turnover as there was an increase in both osteocalcin and CrossLaps. The lack of significance for the last two parameters may be related to the low number of patients. An alternative explanation if turnover should not be increased would be a direct effect on the enzyme bALP of the drug. The observed changes in bone parameters may be associated with CBZ induced disorders of bone metabolism including osteoporosis.

\subsection{1}

EFFECT OF ESLICARBAZEPINE ACETATE ON THE STEADYSTATE PHARMACOKINETICS OF DIGOXIN IN HEALTHY SUBJECTS

Joana Maia, Luis Almeida, Manuel Vaz-da-Silva, Eva Soares, Susana Tavares, Amilcar Falcao, and Patricio Soares-da-Silva (Research \& Development, BIAL, S. Mamede do Coronado, Porto, Portugal)

Rationale: To investigate the effect of eslicarbazepine acetate, a new voltage-gated sodium channel blocker, on the steady-state pharmacokinetics of digoxin.

Methods: ingle-centre, randomized, double-blind, placebocontrolled, two-way crossover study in 12 healthy subjects. The study consisted of two 8-day treatment periods separated by a washout of 10 or more days. On each period, subjects received either a daily oral dose of eslicarbazepine acetate (ESL) $1200 \mathrm{mg}$ once-daily or placebo (PLA) concomitantly with a digoxin once-daily dose of $0.5 \mathrm{mg} /$ day on Days 1 and 2 and $0.25 \mathrm{mg} /$ day on Days 3 to 8 . 
Results: Minimum serum digoxin concentration $\left(\mathrm{C}_{\min }\right)$ at Days 6, 7 and 8 was respectively 445, 452 and $633 \mathrm{pg} / \mathrm{mL}$ with PLA and 475, 522 and $561 \mathrm{pg} / \mathrm{mL}$ with ESL. Serum digoxin concentration-time profile post last digoxin dose (Day 8) in each period is presented in Figure 1. Mean $( \pm \mathrm{SD})$ maximum serum digoxin concentration $\left(\mathrm{C}_{\max }\right)$ and area under the concentration-time curve over the dosing interval $\left(\mathrm{AUC}_{\tau}\right)$ following administration of digoxin with PLA were $2350(1034) \mathrm{pg} / \mathrm{mL}$ and 17607 (5599) pg.h/mL, respectively. Following administration with ESL, mean $\left.( \pm \mathrm{SD}) \mathrm{C}_{\max }\right)$ and $\mathrm{AUC}_{\tau}$ were $1909(596) \mathrm{pg} / \mathrm{mL}$ and 16595 (3801) pg.h/mL, respectively. Digoxin $\mathrm{C}_{\max }$ occurred $\left(\mathrm{t}_{\max }\right)$ at $1 \mathrm{~h}$ post-dose in both periods. Point estimate (PE) and corresponding $90 \%$ confidence intervals $(90 \% \mathrm{CI})$ of the PLA/ESL $\mathrm{C}_{\max }$ geometric mean ratio were 0.85 and $0.68-1.07$. The PLA/ESL digoxin $\mathrm{AUC}_{\tau}$ geometric mean ratio presented a PE of 0.96 and a $90 \% \mathrm{CI}$ of $0.90-1.03$. The $15 \%$ decrease in digoxin $\mathrm{C}_{\max }$, when digoxin was administered concomitantly with ESL, is not expected to affect digoxin efficacy because the extent of exposure (as assessed by $\mathrm{AUC}_{\tau}$ ) was similar and $\mathrm{C}_{\max }$ decrease may reduce the probability of adverse events related with digoxin peak exposure.

Conclusions: Eslicarbazepine acetate, at the dose of $1200 \mathrm{mg}$ oncedaily, has no relevant effect on the steady-state extent of systemic exposure to digoxin.

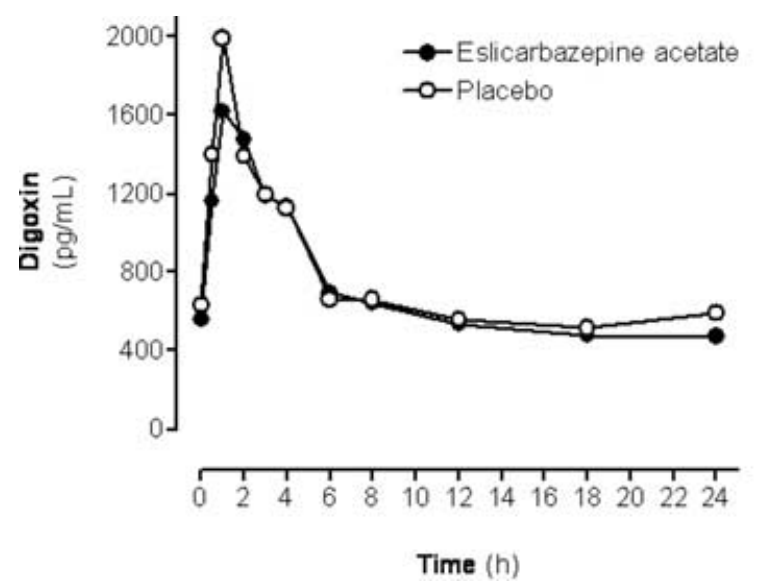

FIG. 1. Mean serum concentration-time profile of digoxin following a 8-day concomitant administration of aslicarbazepine acetate (1200 mg once-daily) and placebo.

\subsection{2}

A PROSPECTIVE STUDY OF THE EFFICACY AND TOLERABILITY OF ZONISAMIDE IN PATIENTS WITH DEVELOPMENTAL DISABILITIES

Alexandra E. McBride, Connie Lau, Rosanne Pachilakis, and Alan B. Ettinger (Neurology, Long Island Jewish Comprehensive Epilepsy Center, New Hyde Park, NY)

Rationale: Zonisamide has proven efficacy and tolerability in treating partial and generalized seizures in the general population. This study will determine response to zonisamide in patients with mental retardation/developmental disabilities and epilepsy. It will assess not only efficacy in reducing seizures but also evaluate the cognitive and behavioral effects of zonisamide in this special population.

Methods: This ongoing prospective observational study recruited adult patients at the outpatient centers of the Long Island Jewish Comprehensive Epilepsy Center who were identified as appropriate candidates for treatment with zonisamide by their epileptologists. Patient's caregivers completed seizure calendars, the Aberrant Behavior Checklist (ABC), Behavioral Problem Inventory (BPI), and Habilitative Improvement Scale (HIS) at baseline, 3 months, 6 months, and 9 months. Zonisamide was titrated to $300 \mathrm{mg}$ total daily over 4 weeks, and it was adjusted further dependent on patient response up to a maximum of $600 \mathrm{mg}$ total daily.

Results: Of 25 patients enrolled, 10 have completed the study. There are 7 females, 3 males. Mean age is 39 years (25-60). Eight patients have a generalized epilepsy syndrome, and two have a partial epilepsy syndrome. The mean number of concomitant anti-epileptic medications is $2.5(1-4)$. Mean total dose of zonisamide reached was $430 \mathrm{mg}$ daily (100-600). Decrease in seizures was seen in 6/10 patients, 3 of whom had $>50 \%$ decrease and $3 \mathrm{had}<50 \%$ decrease. Two patients had no change in seizure frequency, and two patients had an increase in seizures. Given the sample size, $\mathrm{p}$ value of $\mathrm{p}<0.01$ was used to determine significance. On the ABC, significant improvement was found in the domains assessing irritability, lethargy, hyperactivity, and inappropriate speech. The BPI found significant improvement in subsets evaluating the frequency and degree of aggressive behavior. On the HIS, there was overall improvement with a value $\mathrm{p}=0.000$. Side effects included drowsiness in 2 patients, slurred speech in 1 patient, and unsteady gait in 1 patient. One patient developed rash and pancytopenia after taking zonisamide for 8 months.

Conclusions: In the majority of patients, a reduction in seizure frequency was seen. Zonisamide was tolerated by most patients with significant improvement in behavioral domains. These findings indicate that zonisamide is an effective drug in the treatment of seizures while promoting positive behavioral effects in patients with developmental disabilities and epilepsy. (Supported by Eisai.)

\subsection{3}

PATTERNS OF LEVETIRACETAM USE IN THE NEUROSCIENCE ICU (NSICU) SETTING

${ }^{1}$ Jason M. Meckler, ${ }^{1}$ Jerzy P. Szaflrski, ${ }^{1,2}$ Lori A. Shutter, ${ }^{3}$ Magdalena Szaflarski, and ${ }^{1}$ Michael D. Privitera $\left({ }^{1}\right.$ Neurology, University of Cincinnati, Cincinnati, OH; ${ }^{2}$ Neurosurgery, University of Cincinnati, Cincinnati, OH; and ${ }^{3}$ Institute for the Study of Health, University of Cincinnati, Cincinnati, $\mathrm{OH}$ )

Rationale: Patients admitted to NSICU frequently require AEDs for seizure management or prophylaxis. Older AEDs have side effects that decrease their usefulness in ICU. Newer AEDs have overall better side effects and safety profile and are becoming more frequently used in this setting. The goal of this study was to evaluate the patterns of levetiracetam (LEV) use in NSICU.

Methods: We retrospectively reviewed charts of all patients admitted to NSICU at the University Hospital in Cincinnati between 9/1/03 and 9/30/04. We computed descriptive statistics for the entire sample and conducted bivariate tests (Pearson chi-square tests and t-tests) for differences between groups of patients who received or did not receive LEV. We also computed bivariate correlations (Pearson's r) between ICU stay and complications of treatment variables. For the analyses involving the complications of treatment variables, we only included cases where the complication was clearly related to the AEDs and not to the primary disease as judged by the clinician treating the patient.

Results: 552 charts of patients treated in NSICU were reviewed. $358 / 379$ (94.4\%) of NSICU-admitted patients treated with AEDs (ages 16-89) were included in this study. 11 patients were receiving LEV before admission to ICU; therapy with LEV was initiated in 114 patients. A total of 124 patients were treated with LEV in ICU (LEV was discontinued in 1 patient after admission). The 5 most frequent diagnoses were trauma $(\mathrm{n}=104 ; 29.1 \%)$, SAH $(\mathrm{n}=102 ; 28.5 \%)$, ICH $(\mathrm{n}=94$; $26.3 \%)$, brain tumor $(\mathrm{n}=73 ; 20.4 \%)$, and $\mathrm{SDH}(\mathrm{n}=66 ; 18.4 \%)$. Many patients carried multiple diagnoses (e.g., trauma/SAH) and are included in both groups. 110 patients (30.7\%) carried other diagnoses e.g., status epilepticus, CNS infection, or stroke. LEV was more likely to be initiated in patients already taking phenytoin $(\mathrm{p}<0.001)$ and in patients with brain tumors $(p=0.064)$. There was no association between starting LEV and already being treated with carbamazepine or valproic acid ( $p>$ 0.256). Encephalopathy, acute renal failure, and bleeding complications (anemia, thrombocytopenia, coagulopathy) were associated with a prolonged ICU stay $(\mathrm{p}<0.047)$. These complications prompted initiation of LEV more frequently than alternative antiepileptic medications $(\mathrm{p}<$ 0.025 ). Patients with liver failure were more likely to receive LEV ( $\mathrm{p}=$ 0.048).

Conclusions: This study describes the use patterns of LEV in a large sample of patients with various diagnoses who were admitted to the neuroscience ICU. Older patients and patients with previous complications related either to AEDs or underlying disease were preferentially treated with LEV. LEV appears to be a safe alternative to other AEDs in this setting. Further studies of LEV use in ICU are recommended. (Supported by UCB Pharma.) 